\title{
Fractal Image Compression of Satellite Imageries
}

\author{
Veenadevi.S.V \\ Department of Electronics and Communication \\ $\mathrm{R} V$ College of Engineering \\ Bangalore, India
}

\author{
A.G.Ananth \\ Department of Telecommunication \\ $\mathrm{R} V$ College of Engineering \\ Bangalore, India
}

\begin{abstract}
Fractal image coding has the advantage of higher compression ratio, but is a lossy compression scheme. The encoding procedure consists of dividing the image into range blocks and domain blocks and then it takes a range block and matches it with the domain block. The image is encoded by partitioning the domain block and using Affine transformation to achieve fractal compression. The image is reconstructed using iterative functions and inverse transforms. In the present work the fractal coding techniques are applied for the compression of satellite imageries. The compression ratio and Peak Signal to Noise Ratio (PSNR) values are determined for three types of images namely standard Lena image, Satellite Rural image and Satellite Urban image. The Matlab simulation results for the reconstructed image for 4 iterations show that for a compression ratio $\sim 3.2$ and PSNR values achievable for Lena image $\sim 12$, Satellite Rural image $\sim 17.0$ and for Satellite urban image $\sim 22$. Comparison of the present results with the EZW coding indicates that, the fractal compression techniques are found more effective for compression of Satellite Urban imageries since the Satellite Urban images contains more fractals compared to that of Satellite Rural image and Lena image.
\end{abstract}

\section{General Terms}

Study of Fractal image compression

\section{Keywords}

Fractal; quadtree; iterated function system (IFS); image compression

\section{INTRODUCTION}

Arnaud E.Jacquin describes a Fractals have the intrinsic property of having visual complexity while being very low in information content, as they can be described and generated by simple recursive deterministic algorithms [1]. Barnsley suggested [2] that the images can be represented as fractal codes and such representation can be used to achieve high compression [3]. In [4] propose an independent and novel approach to image coding, based on a fractal theory of iterated transformations. The main characteristics of this approach are that it relies on the assumption that image redundancy can be efficiently exploited through self transformability on a block wise basis. Fractal image coding is an efficient image compression technique which is based on the local self similarities within real natural images. The representation of an image by a set of contraction transformation, the fixed point of which is the best possible approximation of the original image $[2,3,4,5]$. The theory of iterated function system (IFS) was developed which are used to describe natural objects [7]. IFS are the mathematical models constructed by finding the parts of images that are affinely similar to the whole image and describe them as reduced copy of whole image [6]. But after iteration it has inferior quality of reconstructed image. In [8] introduced pre classification for fractal compression coding process together with the concept of quartered tree coding. Improves the coding time, compression ratio but PSNR suffers a little. The encoding time of fractal compression technique is too long to achieve image compression [9] suggest improved algorithm form the aspect of image segmentation. The fractal image compression the encoding step is computationally expensive, because every range block must be compared to all domain blocks in the codebook to find the best-matched one during the coding procedure. In [10] fast classification algorithm using DCT coefficients is proposed. Simulation results show that the runtime of the proposed algorithm is reduced greatly compared to the existing methods. At the same time, the new algorithm also achieved high PSNR values. Fractal coding is a novel method to compress images. It offers many advantages. In [11] proposes a new method using best polynomial approximation to decide whether a domain block is similar enough to a given range block. Also gives a kind of domain pool. It is found that the probability distribution of 8 isometrics in the fractal code is not average. And consequently it is proposed to use only 2 or 4 isometrics to speed up compression. In [12] propose three algorithms for fractal image decoding in which the decoding will be done in less iteration, so it would be faster. Then in decompression, we substitute the pixels of each range with the average of their corresponding domain. Which provides a very high quality image at almost one iteration without any change in compression ratio.

\section{FRACTAL COMPRESSION}

There are several different ways to approach the fractal compression. One way is to use the fixed point transformation. A function $f($.$) is said to have a fixed point x_{0}$ if $f\left(x_{0}\right)=x_{0}$. Suppose the function $f($.) to be of the form $a x+b$. Then, except for when $\mathrm{a}=1$, this equation always has a fixed point.

$a x_{0}+b=x_{0}$ then $x_{0}=b /(1-a)$

This means that to transmit the value of $x_{0}$, using the values of ' $a$ ' and ' $b$ ' and obtain $x_{0}$ at the receiver using (1). Instead of solve this equation to obtain $\mathrm{x}_{0}$, we could take a guess at what $\mathrm{x}_{0}$ using recursion

$x_{0}(n+1)=a x_{0}(\mathrm{n})+b$

Thus, the value of $x_{0}$ is accurately specified by fixed point equation. The receiver can retrieve the value either $b$ the 
solution of (1) or via the recursion (2). To generalize this idea, suppose that for a given image $\zeta$ treated as an array of integers, exists a function $\mathrm{f}($.$) such that \mathrm{f}(\zeta)=\zeta$. Then $\mathrm{f}($.$) as the$ compressed representation of $\zeta$. This idea was first proposed by Michael and Alan Sloan based on the idea of self similarity and natural objects can be obtained as the fixed point of a certain type of function. Instead of generating a single function directly for which the given image is a fixed point. We partition the image into blocks $R_{k}$, called range blocks, and obtain a transformation $f_{k}$ for each block. The transformations $f_{k}$ are not fixed point transformations since they do not satisfy the equation $f_{k}\left(R_{k}\right)=R_{k}$. Instead, they are a mapping from a block of pixels $D_{k}$ from some other part of the image. While each individual mapping $f_{k}$ is not a fixed point mapping, that can combine all these mappings to generate a fixed point mapping. The image blocks $D_{k}$ are called domain blocks, and they are chosen to be larger than the range blocks. The domain blocks are obtained by sliding a K X K window over the image in steps of $\mathrm{K} / 2$ or $\mathrm{K} / 4$ pixels. As long as the window remains within the boundaries of the image. The transformations $\mathrm{f}_{\mathrm{k}}$ are composed of a geometric transformation $\mathrm{g}_{\mathrm{k}}$ and a massic transformation $\mathrm{m}_{\mathrm{k}}$. The geometric transformation consists of moving the domain block to the location of the range block and adjusting the size of the domain block to match the size of the range block.

$$
\breve{R}_{k}=f_{k}\left(D_{k}\right)=m_{k}\left(g_{k}\left(D_{k}\right)\right)
$$

$\breve{\mathrm{R}}_{\mathrm{k}}$ instead of $\mathrm{R}_{\mathrm{k}}$ in (3) because it is not possible to find an exact functional between domain and range blocks, since some loss of information. This loss is measured in terms of mean squared error. In order to reduce the computations, restrict the number of domain blocks to search. However, in order to get the best possible approximation, the pool of domain blocks to be as large as possible. The elements of the domain pool are then divided in to shade blocks, edge blocks, and midrange blocks. The shade blocks are those in which the variance of pixel values within the block is small. The edge block, contains those blocks that have a sharp change of intensity values. The midrange blocks are those that fit into not too smooth but with no well defined edges. The encoding procedure proceeds as a range block is classified into one of the three categories described above. If it is a shade block, send the average value of the block. If it is a midrange block, the massic transformation is of the form $\left(\alpha_{k} t_{i j}+\Delta_{k}\right)$ where $T_{k}=g_{k}\left(D_{k}\right)$, and $t_{i j}$ as the ij ${ }^{\text {th }}$ pixel in $T_{k} i, j=0,1, \ldots$. $\mathrm{M}-1, \alpha_{\mathrm{k}}$ is selected from a small set of values. Thus the possible values of $\alpha$ and the midrange domain blocks in the domain pool in order to find the $\left(\alpha_{k}, D_{k}\right)$ pair that will minimize $d\left(R_{k}, \alpha_{k}\right.$ $\left.T_{k}\right)$. The value of $\Delta_{k}$ is then selected as the difference of the average values of $R_{k}, \alpha_{k} T_{k}$. If the range block $R_{k}$ is classified as an edge block. The block is first divided into a bright and a dark region. The dynamic range of the block $r_{d}\left(R_{k}\right)$ is the computed as the difference of the average values of the light and dark regions. For a given domain block, then used to compute the value of $\alpha_{k}$ by

$\alpha_{k}=\min \left\{\left(r_{d}\left(R_{k}\right)\right) /\left(r_{d}\left(T_{k}\right)\right), \alpha_{\max }\right\}$

In (4) where $\alpha_{\max }$ is an upper bound on the scaling factor.

The work carried out in the paper starts from basic fractal image coding based on quadtree. Taking different images such as standard Lena, satellite Rural and satellite Urban images of size
$128 \times 128$ pixels for fractal compression. The Compression ratio and PSNR is calculated for the reconstructed image for the fixed Range block of size 4 X 4 .

\section{QUADTREE APPROACH}

A quadtree partition is a representation of an image as a tree in which each node, corresponding to a square portion of the image, contains four sub-nodes, corresponding to the four quadrants of the square. The root of the tree is the initial image. Decompose an image based on this approach is first assign the whole image to the root of the tree and examine its uniformity. If the uniformity criterion is not met, it is quartered into four sub images. These four sub images have the same size and are associated with the four child nodes of the root. The uniformity of each sub image will be examined next, and the sub image will be subdivided repeatedly until the uniformity criterion is met or some minimum sub image size has been reached [7].

\subsection{Affine transformation}

An affine transformation $\mathrm{w}: \mathrm{Rn} \rightarrow \mathrm{Rn}$ can always be written as $\mathrm{w}=\mathrm{Ax}+\mathrm{b}$, where $\mathrm{A} € \mathrm{Rn} \times \mathrm{n}$ is an $\mathrm{n} \times \mathrm{n}$ matrix and $\mathrm{b} € \mathrm{Rn}$ is an offset vector. Such transformation will be contractive exactly when its linear part is contractive, and this depends on the metric used to measure distances. A linear transformation can scale with As, stretch with At, skew with $\mathrm{Au}$, and rotate with $\mathrm{A} \theta$.

\subsection{Iterated function system (IFS)}

The IFS model generates a geometrical shape with an iterative process. An IFS based modeling system is defined by a triple (x, $\mathrm{d}, \mathrm{s})$ where $(\mathrm{x} \mathrm{d})$ is a complete metric space, $\mathrm{x}$ is called iterative space. $S$ is a semi group acting on points of $x$ such that $p \rightarrow T p$ where $T$ is a contractive operator, $s$ is called iterative semi group. An IFS I is a finite subset of $\mathrm{s}$ : $\mathrm{I}=\left\{\mathrm{T}_{0}, \mathrm{~T}_{1}, \mathrm{~T}_{\mathrm{N}-1}\right\}$ with operators $\mathrm{Ti} € \mathrm{~s}{ }^{8}$.

\section{THE PROPOSED ALGORITHM}

The algorithm steps are as follows.

1. Divides the original image into Range block size, do not overlap. Taking of fixed dimension of Range block as $4 \times 4$.

2. Taken Domain block $D_{i}$ twice the size of the Range block $R_{i}$ in the original image.

3. Partitioning the Domain blocks doing scaling, averaging, rotation and calculating contrast that is affine transformations $\mathrm{D}_{\mathrm{i}}{ }_{\mathrm{i}}$ to Domain block $\mathrm{D}_{\mathrm{i}}$

4. Calculate the root of mean square of Range block $R_{i}$ and each corresponding transformed Domain block $\mathrm{D}^{1}{ }_{\mathrm{i}}$, as the matching error $\mathrm{d}$ between the two blocks. If the matching error to satisfy $d<\epsilon, \epsilon$ is a present tolerable error, skip to step6.

5. If a full search completed, and did not meet the conditions $d<\epsilon$, segment the original block $R_{i}$ into four equal, repeat (2) to (6) operations.

6. Record the fractal coding information to complete a fractal encoding.

7. For the encoding image applying 4 iterations and inverse transform to reconstruct the image and calculating PSNR and Compression ratio 


\section{RESULTS AND DISCUSSIONS}

The algorithm realized in Matlab to code and to decode the satellite image of Urban of size 1377 X 955 , Rural image of size 995 X 571 and Lena image of size 256 X 256. But all these images are resized to $128 \times 128$. Experimental parameters are listed as Range block of size is 4, 8, 16 and number of iterations. One can see that better PSNR by choosing fixed Range block of size 4, otherwise PSNR decreases. The number of iteration is taken as 4 for all three images. Increased iteration is found to affect the quality on the image. The compression ratios and the PSNR values obtained for the reconstructed Lena Image, Satellite Rural Image and Satellite Urban Image is listed in Table 1. The original image and the reconstructed image after fractal encoding- decoding is shown in Figure 1, 2 and Figure 3.

It is clearly seen from the Table 1 that for a compression ratio $\sim 3.2$ the PSNR values achievable for Lena image, Satellite Rural Image and Satellite Urban image are different. The Lena image shows the lowest PSNR $\sim 11.91$ and the Rural image $\sim 17.09$ and the Urban image $\sim 21.89$. The Urban image shows the highest PSNR values compared to Rural and Lena image. Further it can be seen from the Figures that the reconstructed Urban image has a better quality of the reconstructed image compared to that of Rural and Lena image. These results suggest that the Urban images contain more fractal information compared to that of Rural or Lena image. The fractal coding techniques are better suited for the compression of satellite Urban images.

TABLE 1. THE EXPERIMENTAL DATA

\begin{tabular}{|l|l|l|l|l|}
\hline $\begin{array}{l}\text { Test } \\
\text { images }\end{array}$ & $\begin{array}{l}\text { Range } \\
\text { Block } \\
\text { of size }\end{array}$ & $\begin{array}{l}\text { No. of } \\
\text { Iterations }\end{array}$ & $\begin{array}{l}\text { Compression } \\
\text { ratio }\end{array}$ & $\begin{array}{l}\text { PSNR in } \\
\mathrm{dB}\end{array}$ \\
\hline $\begin{array}{l}\text { Lena } \\
\text { image }\end{array}$ & 4 & 4 & 3.2 & 11.91 \\
\hline $\begin{array}{l}\text { Satellite } \\
\text { Rural } \\
\text { image }\end{array}$ & 4 & 4 & 3.2 & 17.09 \\
\hline $\begin{array}{l}\text { Satellite } \\
\text { Urban } \\
\text { image }\end{array}$ & 4 & 4 & 3.2 & 21.89 \\
\hline
\end{tabular}

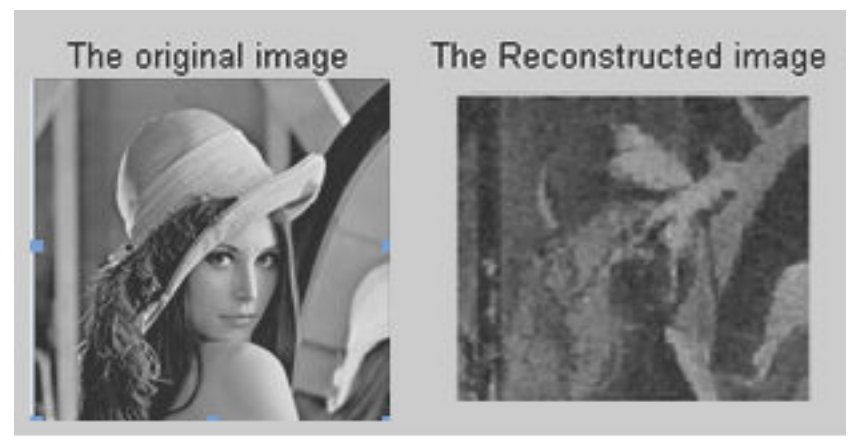

Figure 1. Lena image

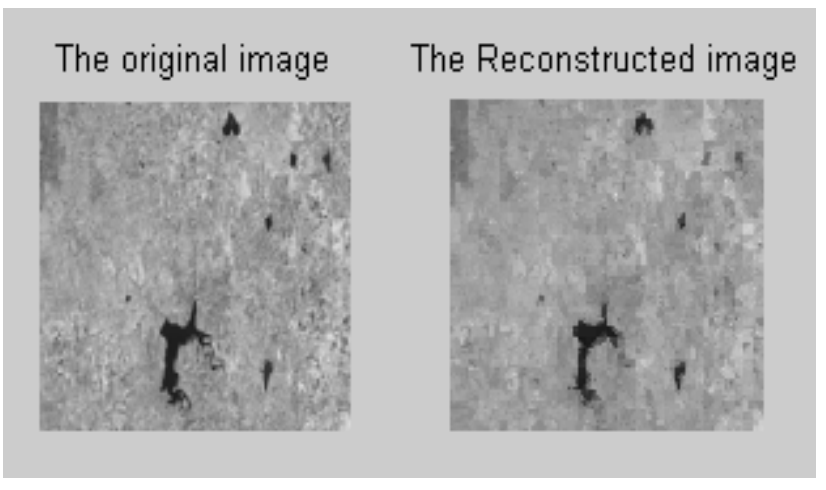

Figure 2. Satellite Rural image

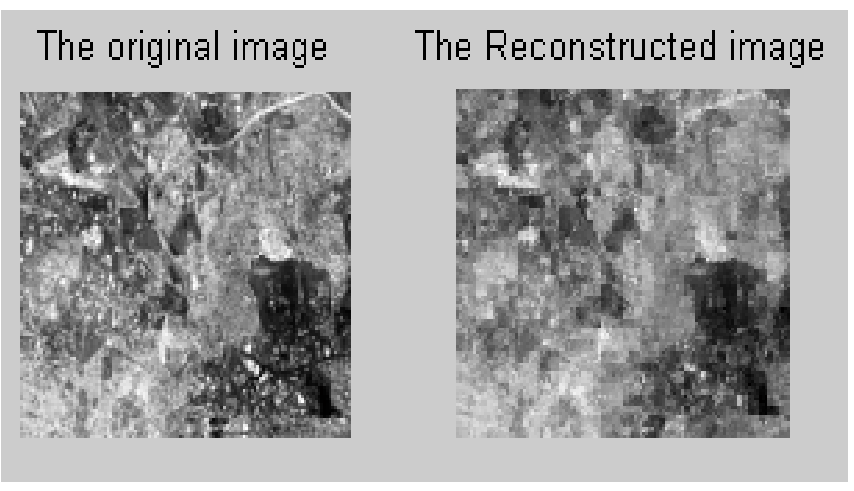

Figure 3. Satellite Urban image

Table 2 shows a comparison of the compression ratios and PSNR derived for the Lena, Satellite Rural and Urban images derived using Fractal and EZW compression methods [13]. It is evident from the Table that for the standard Lena image, the EZW coding shows higher Compression ratios and PSNR values compared to fractal techniques indicating that the Lena image is do not contain significant fractals.

TABLE 2. The Compression ratios and PSNR obtained form Embedded Zero Tree Wavelet (EZW) coding and Fractal coding

\begin{tabular}{|c|c|c|c|c|}
\hline \multirow{2}{*}{$\begin{array}{l}\text { Test } \\
\text { images }\end{array}$} & \multicolumn{2}{|c|}{ Fractal Compression } & \multicolumn{2}{|c|}{ EZW Compression } \\
\hline & $\begin{array}{l}\text { Compression } \\
\text { ratio }\end{array}$ & $\begin{array}{l}\text { PSNR } \\
\text { in } d B\end{array}$ & $\begin{array}{l}\text { Compression } \\
\text { Ratio }\end{array}$ & $\begin{array}{l}\text { PSNR } \\
\text { in } d B\end{array}$ \\
\hline $\begin{array}{l}\text { Lena } \\
\text { image }\end{array}$ & 3.2 & 11.91 & 11.28 & 27.65 \\
\hline $\begin{array}{l}\text { Satellite } \\
\text { Rural } \\
\text { image }\end{array}$ & 3.2 & 17.09 & 5.75 & 22.24 \\
\hline $\begin{array}{l}\text { Satellite } \\
\text { Urban } \\
\text { image }\end{array}$ & 3.2 & 21.89 & 1.39 & 9.26 \\
\hline
\end{tabular}


Whereas for the satellite Rural image the EZW coding shows higher compression ratios and PSNR values compared to Fractal coding indicating that the Rural Images do not contain significant fractals and the reconstructed image form the fractal coding will be of poor quality due to low PSNR Values. However for the urban images the Fractal show higher compression ratios and PSNR compared to EZW techniques indicating the presence of significant fractals in the satellite Urban image. This suggests that the Fractal coding is extremely suited for compressing the Satellite Urban images to produce better quality of reconstructed images with higher PSNR values.

\section{CONCLUSIONS}

From the analysis carried out in the paper the following conclusions can be drawn

1. The fractal encoding techniques can be applied for achieving high compression ratios and better PSNR values for satellite Imageries.

2. For satellite Urban images one can achieve high compression ratios $\sim 3.2$ with PSNR values $\sim 22$ compared to the satellite Rural images

3. The Urban images contains more fractal information compared to that of Rural and Lena images.

4. The fractal compression techniques are more applicable to Satellite Urban images compared to satellite Rural images and standard Lena images.

5. The fractal coding is applicable where the presence of man made artificial Urban features manifest itself in the form fractals in satellite urban imageries.

6. Further improvement in the Compression ratio and the PSNR values for the fractal coding can be achieved by considering the different dimensions of the Range Blocks and optimizing number of iteration.

\section{REFERENCES}

[1] Arnaud E. Jacquin, "Fractal image coding", Proceedings of IEEE VOL.81, pp. 1451-1465, 1993.

[2] Barnsley MF. " Fractal everywhere", $2^{\text {nd }}$ ed, San Deigo Academic Press, 1993.

[3] M.F Barnsley, Alan d.Salon, "Better way to compress images', 1993.
[4] Arnaud E. Jacquin A, "Image coding based on a fractal theory of iterated contractive image transformations", IEEE Trans Image processing , 1992, pp. 18-30

[5] Fisher Y, editor, "Fractal image compression: theory and application”, New York, Springer-Verlag, 1995.

[6] Dr. Muhammad Kamran, Amna Irshad Sipra and Muhammd Nadeem, "A novel domain optimization technique in fractal image compression", IEEE Proceedings of the 8th World Congress on Intelligent Control and Automation, 2010, pp. 994-999.

[7] Bohong Liu and Yung Yan, "An Improved Fractal Image Coding Based on the Quadtree", IEEE 3rd International Congress on Image and Signal Processing, 2010,pp. 529532

[8] Kai Shuang, Ning Xiao, Feng $\mathrm{Xu}$, Dayue Lv and Wang $\mathrm{Yu}$, " Fractal Compression Coding based on Fractal Dimension Feature Blocks", IEEE International Symposium on Information Science and Engineering, 2008, pp. 223-226.

[9] Hui Yu, Li Li, Dan Liu, Hongyu Zhai, Xiaoming Dong, “ Based on Quadtree Fractal Image Compression Improved Algorithm for Research", IEEE Trans, 2010, pp.1-3.

[10] Chelehgahi, Mehdi Masoudi, Jaferzadeh, Keyyan, Nia, Mohsen Derakhshan, “ A high speed intelligent classification algorithm for fractal image compression using DCT coefficients", IEEE $3^{\text {rd }}$ International conference on ICCSN, May 2011, pp.156-159.

[11] Zhuang Wu, Bixi Yan, "An effective fractal image compression algorithm" IEEE International conference on ICCASM, 2010, pp.139-143.

[12] Hosseini, Shookooh, Shahhosseini, Beizaee, "Speeding up fractal image de-compression", IEEE International conference on ICCAIE, 2010, pp.521-526.

[13] K. Nagamani and A.G.Ananth, "Study of embedded zero tree wavelet (EZW) compression techniques for high and low resolution satellite imageries", International Journal of Technology and Engineering System, 2010, pp. 141-145. 